\title{
Deficiency of tenascin-C and attenuation of blood-brain barrier disruption following experimental subarachnoid hemorrhage in mice
}

\author{
Masashi Fujimoto, MD, PhD, ${ }^{1}$ Masato Shiba, MD, PhD, ${ }^{1}$ Fumihiro Kawakita, MD, ${ }^{1}$ Lei Liu, MD, ${ }^{1}$ \\ Naoshi Shimojo, PhD, 2,3 Kyoko Imanaka-Yoshida, MD, PhD, , ${ }^{2,3}$ Toshimichi Yoshida, MD, PhD, ${ }^{2,3}$ and \\ Hidenori Suzuki, MD, PhD1,3
}

Departments of ${ }^{1}$ Neurosurgery and ${ }^{2}$ Pathology and Matrix Biology, ${ }^{3}$ Research Center for Matrix Biology, Mie University Graduate School of Medicine, Tsu, Japan

OBJECTIVE Tenascin-C (TNC), a matricellular protein, is induced in the brain following subarachnoid hemorrhage (SAH). The authors investigated if TNC causes brain edema and blood-brain barrier (BBB) disruption following experimental SAH.

METHODS C57BL/6 wild-type (WT) or TNC knockout (TNKO) mice were subjected to SAH by endovascular puncture. Ninety-seven mice were randomly allocated to WT sham-operated $(n=16)$, TNKO sham-operated $(n=16)$, WT SAH $(n=$ $34)$, and TNKO SAH ( $n=31)$ groups. Mice were examined by means of neuroscore and brain water content 24-48 hours post-SAH; and Evans blue dye extravasation and Western blotting of TNC, matrix metalloproteinase (MMP)-9, and zona occludens (ZO)-1 at 24 hours post-SAH. As a separate study, 16 mice were randomized to WT sham-operated, TNKO sham-operated, WT SAH, and TNKO SAH groups ( $n=4$ in each group), and activation of mitogen-activated protein kinases (MAPKs) was immunohistochemically evaluated at 24 hours post-SAH. Moreover, 40 TNKO mice randomly received an intracerebroventricular injection of TNC or phosphate-buffered saline, and effects of exogenous TNC on brain edema and BBB disruption following SAH were studied.

RESULTS Deficiency of endogenous TNC prevented neurological impairments, brain edema formation, and BBB disruption following SAH; it was also associated with the inhibition of both MMP-9 induction and ZO-1 degradation. Endogenous TNC deficiency also inhibited post-SAH MAPK activation in brain capillary endothelial cells. Exogenous TNC treatment abolished the neuroprotective effects shown in TNKO mice with SAH.

CONCLUSIONS Tenascin- $C$ may be an important mediator in the development of brain edema and BBB disruption following SAH, mechanisms for which may involve MAPK-mediated MMP-9 induction and ZO-1 degradation. TNC could be a molecular target against which to develop new therapies for SAH-induced brain injuries.

http://thejns.org/doi/abs/10.3171/2015.4.JNS15484

KEY WORDS tenascin-C; brain edema; blood-brain barrier disruption; early brain injury; subarachnoid hemorrhage; knockout mice; vascular disorders

$\mathrm{A}$ NEURYSMAL subarachnoid hemorrhage (SAH) is one of the most life-threatening cerebrovascular disorders, with high mortality and morbidity rates. ${ }^{26,33}$ Delayed cerebral ischemia (DCI) remains the most important cause of morbidity and mortality in those patients who survive the initial bleeding. ${ }^{20}$ Recent studies have reported that early brain injury (EBI), as well as cerebral vasospasm, is a major cause of DCI following SAH..$^{20}$ EBI occurs before the onset of cerebral vasospasm and may cause DCI with no significant vasospasm. ${ }^{3}$ Brain edema, which results mainly from blood-brain barrier (BBB) disruption, ${ }^{18}$ plays an important role in the pathological

ABBREVIATIONS BBB = blood-brain barrier; $\mathrm{DCI}$ = delayed cerebral ischemia; EBI = early brain injury; ERK = extracellular signal-regulated kinase; ICA = internal carotid artery; JNK = c-Jun N-terminal kinase; MAPK = mitogen-activated protein kinase; MMP = matrix metalloproteinase; PBS = phosphate-buffered saline; PDGF = plateletderived growth factor; SAH = subarachnoid hemorrhage; TNC = tenascin-C; TNKO = TNC knockout; WT = wild-type; ZO = zona occludens.

SUBMITTED March 2, 2015. ACCEPTED April 20, 2015.

INCLUDE WHEN CITING Published online October 16, 2015; DOI: 10.3171/2015.4.JNS15484. 
manifestation of EBI following SAH. ${ }^{20}$ However, the molecular mechanisms of brain edema formation and BBB disruption following SAH remain poorly understood.

Tenascin-C (TNC), a matricellular protein, is induced in response to injury and exerts diverse functions. ${ }^{13}$ As with other matricellular protein knockout mice, TNC knockout (TNKO) mice undergo normal development and display no distinct morphological phenotypes..$^{21}$ However, many studies using TNKO mice have reported that TNC may play an important role in aggravating or improving various diseases. ${ }^{9,17}$

In the CNS, TNC is highly expressed in astrocytes during early stages of development, whereas its expression is markedly low in the normal adult CNS.${ }^{6}$ Recently, it was reported that TNC was induced in CSF and serum following aneurysmal SAH in a clinical setting. ${ }^{33,31}$ The CSF TNC levels were significantly higher in patients with poor outcome than in those with good outcome. ${ }^{31}$ An experimental study also showed that TNC was induced in the cerebral cortex following SAH in rats. ${ }^{25}$ To date, however, there is no direct evidence showing that TNC causes brain injury. Accumulating evidence suggests a role for matrix metalloproteinase (MMP)-9 in the early disruption of the BBB following $\mathrm{SAH},{ }^{20}$ and TNC upregulates MMP9 expression in certain situations..$^{12,14,15}$ Therefore, in the present study, we used TNKO mice to evaluate the role of TNC induction in brain edema formation and BBB disruption following SAH.

\section{Methods \\ Animals}

All procedures were approved by the Animal Ethics Review Committee of Mie University and were carried out in accordance with the institution's Guidelines for Animal Experiments. The original TNKO mouse was backcrossed with C57BL/6 inbred mice for more than 10 generations. ${ }^{21}$ C57BL/6 wild-type (WT) littermates were used as controls. Mice were maintained on a constant 12-hour light/12-hour dark cycle in a temperature- and humidity-controlled room and were given ad libitum access to food and water. All mice used in the experiments were female.

\section{Subarachnoid Hemorrhage Model and Study Protocol}

The endovascular perforation model of SAH was produced by a modification of the method previously described. ${ }^{1}$ Each animal was anesthetized with an intraperitoneal injection of tribromoethanol $(250 \mu \mathrm{g} / \mathrm{g}$ body weight). A sharpened 5-0-monofilament nylon suture was advanced rostrally into the left internal carotid artery (ICA) from the external carotid artery stump to perforate the bifurcation of the left anterior and middle cerebral arteries. In the sham surgery, the filament was advanced 5 $\mathrm{mm}$ through the ICA without perforating the artery. Blood pressure and heart rate were measured noninvasively from the tail.

First, to study the effects of endogenous TNC induction following $\mathrm{SAH}$ on brain edema formation and $\mathrm{BBB}$ disruption, $50 \mathrm{WT}$ and 47 TNKO mice (weight 20-25 g) were randomly divided into WT sham-operated $(\mathrm{n}=16)$,
TNKO sham-operated $(\mathrm{n}=16)$, WT SAH $(\mathrm{n}=34)$, and TNKO SAH $(\mathrm{n}=31)$ groups. Mice underwent endovascular perforation SAH or sham operation. After neuroscore was evaluated, mice were euthanized $24-48$ hours postsurgery. High-resolution pictures of the base of the brain depicting the circle of Willis and basilar arteries were taken to assess the severity of SAH. The time points were selected because previous studies reported that post-SAH BBB disruption peaked within 24 hours and was reversed by $48-72$ hours following experimental SAH. ${ }^{23,29}$ The brain water content was measured at 24 and 48 hours postsurgery, and BBB permeability and Western blot analyses were evaluated at 24 hours postsurgery (Fig. 1 upper).

As a separate study, WT and TNKO mice were randomized to WT sham-operated $(\mathrm{n}=4)$, TNKO shamoperated $(n=4)$, WT SAH $(n=4)$, and TNKO SAH (n = 4) groups. After assessing SAH severity, activation of mitogen-activated protein kinases (MAPKs) was immunohistochemically evaluated at 24 hours postsurgery.

Next, to study the effects of an exogenous TNC injection on TNKO mice with SAH, 40 TNKO mice randomly underwent $\mathrm{SAH}$ or sham operation, and $5 \mu \mathrm{g}$ of TNC or phosphate-buffered saline (PBS; vehicle) was administered intracerebroventricularly at 30 minutes postsurgery. Neuroscore, SAH severity, brain water content, and BBB permeability were evaluated at 24 hours postsurgery (Fig. 1 lower).

\section{Tenascin-C Preparation and Intracerebroventricular Infusion}

Tenascin- $\mathrm{C}$ was purified from culture supernatant of the U-251MG glioma cell line by ammonium sulfate precipitation, Sephacryl S-500 gel filtration, Mono Q ionexchange chromatography, and using a hydroxyapatite column as previously described. ${ }^{35}$ Purified TNC (purity $>95 \%$ ) was extensively dialyzed against $\mathrm{Ca}^{2+}, \mathrm{Mg}^{2+}$-free Dulbecco's PBS and passed through a $0.2-\mu \mathrm{m}$ membrane filter. The $\mathrm{pH}$ of the TNC solution was maintained within the physiological range of 7.2-7.6. Regarding dosage, we estimated that the calculated concentration was equivalent to the CSF concentration of TNC reported in patients with $\mathrm{SAH}^{32}$ when 1-10 $\mu \mathrm{g}$ of TNC was injected into the cisterna magna in rats in our previous study, in which TNC caused no brain injury. ${ }^{8}$ Because the CSF volume of mice is considered to be $1 / 4-1 / 9$ of that of rats, less than $2.5 \mu \mathrm{g}$ of TNC injection into the CSF may be enough to obtain an equivalent CSF TNC concentration in mice in a clinical setting. ${ }^{36}$ However, we selected a higher dose of TNC $(5 \mu \mathrm{g})$, because TNC was induced in the brain following $\mathrm{SAH}$. Therefore, the brain concentration of TNC would be much higher than the reported CSF concentration in a clinical setting. ${ }^{24}$

The intracerebroventricular infusion was performed by a modification of the method previously described. ${ }^{1}$ The needle of a 2- $\mu$ l Hamilton syringe (Hamilton Co.) was inserted through a bur hole into the left lateral ventricle using the following coordinates relative to the bregma: $0.2 \mathrm{~mm}$ posterior, $1.0 \mathrm{~mm}$ lateral, and $2.2 \mathrm{~mm}$ below the horizontal plane of the bregma. PBS $(1 \mu \mathrm{l})$ or TNC $(5 \mu \mathrm{g}$ in $1 \mu \mathrm{l}$ of PBS) was infused at a rate of $0.5 \mu \mathrm{l} /$ minute (irrespective of the animal's body weight) at 30 minutes after 
Experiment $1: 4$ groups (97 mice)

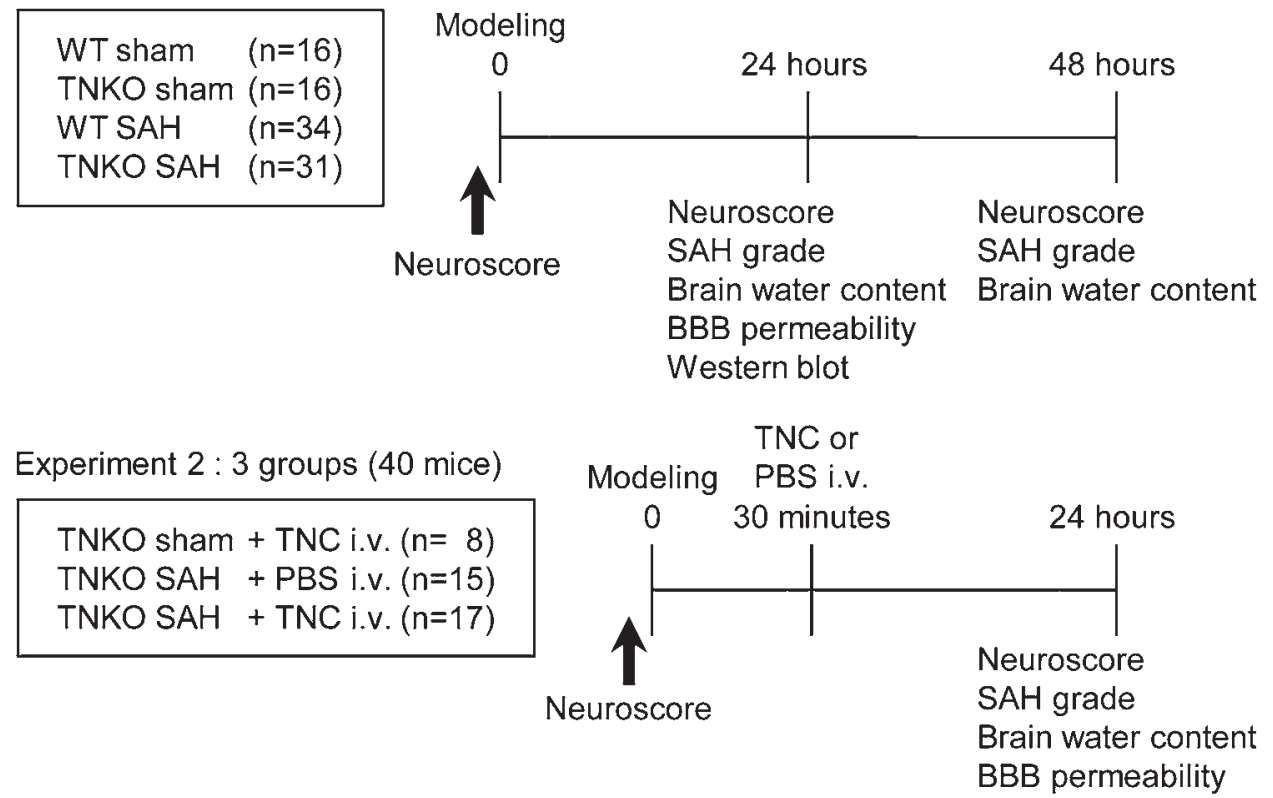

FIG. 1. Experimental designs. Experiments 1 (upper) and 2 (lower) were designed to examine the effects of endogenous and exogenous TNC on brain edema and BBB disruption following SAH, respectively. i.v. = intracerebroventricular injection.

surgery. The needle was removed 10 minutes after an infusion, and the wound was quickly sutured.

\section{Neurobehavioral Test}

Neurological impairments were blindly evaluated using 2 methods. Neurological scores (3-18) were assessed by summing 6 test scores (spontaneous activity; spontaneous movement of 4 limbs; forepaw outstretching; climbing; body proprioception; and response to whisker stimulation), as previously described. ${ }^{8} \mathrm{~A}$ beam balance test investigated the animal's ability to walk on a narrow wooden beam for 60 seconds: 4 points, walking $\geq 20 \mathrm{~cm} ; 3$ points, walking $\geq 10 \mathrm{~cm}$ but $<20 \mathrm{~cm} ; 2$ points, walking $\geq 10 \mathrm{~cm}$ but falling; 1 point, walking $<10 \mathrm{~cm}$; and 0 points, falling while walking $<10 \mathrm{~cm}$. The median score of 3 consecutive trials in a 5-minute period was calculated.

\section{Severity of SAH}

Because the severity of brain injuries in the endovascular perforation model of SAH is correlated with the severity of $\mathrm{SAH}, 2,27$ the $\mathrm{SAH}$ grading score was used for the standardization of brain injuries. The severity of SAH was blindly assessed using the high-resolution photographs as previously described., ${ }^{1,27}$ The basal cistern was divided into 6 segments, and each segment was allotted a grade from 0 to 3 , depending on the amount of subarachnoid blood clot in the segment: Grade 0 , no subarachnoid blood; Grade 1, minimal subarachnoid blood; Grade 2, moderate blood clot with recognizable arteries; and Grade 3 , blood clot obliterating all arteries within the segment. The animals received a total score ranging from 0 to 18 after adding the scores from all 6 segments.

\section{Brain Water Content}

Brain edema was determined using the wet/dry method as previously described. ${ }^{1}$ After euthanizing mice under deep anesthesia, brains were quickly removed and separated into the left and right cerebral hemispheres, cerebellum, and brainstem, and then weighed (wet weight). Brain specimens were dried in an oven at $105^{\circ} \mathrm{C}$ for 72 hours and weighed again (dry weight). The percentage of water content was calculated according to the following formula: ([wet weight - dry weight $] /$ wet weight $) \times 100 \%$.

\section{Blood-Brain Barrier Permeability}

Blood-brain barrier permeability was assessed by measuring the extravasation of Evans blue dye as previously described. ${ }^{1}$ At 24 hours postsurgery, Evans blue dye (2\%; $5 \mathrm{ml} / \mathrm{kg}$ body weight) was injected intraperitoneally and allowed to circulate for 3 hours. Under deep anesthesia, mice were euthanized by intracardial perfusion with PBS, and brains were removed and divided into the same regions as in the brain water content study. Brain specimens were weighed, homogenized in $0.7 \mathrm{ml}$ of PBS, and centrifuged at $15,000 \mathrm{~g}$ for 30 minutes. Then, $0.5 \mathrm{ml}$ of the resultant supernatant was added to an equal volume of trichloroacetic acid. After overnight incubation at $4^{\circ} \mathrm{C}$ and centrifugation at $15,000 \mathrm{~g}$ at $4^{\circ} \mathrm{C}$ for 30 minutes, the supernatant was taken for spectrophotometric quantification of extravasated Evans blue dye at $610 \mathrm{~nm}$.

\section{Western Blot Analyses}

Western blot analyses were performed as previously described. ${ }^{8}$ In the present study, the left (perforation 
side) cerebral hemisphere was used. Equal amounts of protein samples $(5 \mu \mathrm{g})$ were loaded on sodium dodecyl sulfate-polyacrylamide gel electrophoresis (SDS-PAGE) gels, electrophoresed, and transferred onto a polyvinylidene difluoride membrane. The membranes were blocked with $5 \%$ bovine serum albumin, followed by incubation overnight at $4{ }^{\circ} \mathrm{C}$ with the rabbit polyclonal anti-TNC antibody (1 $\mu \mathrm{g} / \mathrm{ml}$; Immuno-Biological Laboratories), rabbit polyclonal anti-MMP-9 antibody (1:1000; Millipore Biosciences Research Reagents SBU), and rabbit polyclonal anti-zona occludens (ZO)-1 antibody (1:1000; Invitrogen Corp.). Blot bands were detected with a chemiluminescence reagent kit (ECL Prime; Amersham Bioscience) and quantified by densitometry with ImageJ software (National Institutes of Health). Beta-tubulin (1:2000; Santa Cruz Biotechnology) was blotted onto the same membrane as a loading control.

\section{Immunohistochemical Staining}

Immunohistochemical staining was performed as previously reported. ${ }^{24}$ At 24 hours postsurgery, mice were euthanized under deep anesthesia with intraperitoneal tribromoethanol and perfused using a cardiac catheter with $50 \mathrm{ml}$ PBS followed by 15 minutes of $10 \%$ neutral buffered formalin at $60-80 \mathrm{~mm} \mathrm{Hg}$. The brains were removed and high-resolution pictures of the base of the brain were taken to assess the severity of SAH. Then, the brains were fixed in $10 \%$ neutral buffered formalin for approximately 12 hours at $4^{\circ} \mathrm{C}$ and embedded in paraffin. Four-micronthick coronal sections at $1.0 \mathrm{~mm}$ posterior to bregma were cut. After dewaxing and rehydration, the sections were placed in 1 mmol EDTA (pH 8.0) and heated in an autoclave at $80^{\circ} \mathrm{C}$ for 20 minutes to retrieve antigen. The sections were incubated in $1 \%$ hydrogen peroxide for $10 \mathrm{~min}$ utes to quench any endogenous peroxidase activity. The sections were blocked with 5\% goat or horse serum. They were then incubated overnight at $4^{\circ} \mathrm{C}$ with the rabbit polyclonal antiphosphorylated extracellular signal-regulated kinase (ERK)1/2, mouse monoclonal antiphosphorylated c-Jun N-terminal kinase (JNK), and mouse monoclonal antiphosphorylated p38 (1:200; Santa Cruz Biotechnology) antibodies. The sections were subsequently incubated with biotinylated antirabbit or antimouse immunoglobulin (Vector Laboratories) for 30 minutes and then with an avidin-biotin complex for 30 minutes at room temperature. Color reactions were developed in diaminobenzidine/hydrogen peroxide solution and the sections were counterstained with hematoxylin solution for light microscopic examination. Negative controls consisted of serial sections incubated with buffer alone instead of the primary antibodies. Hematoxylin and eosin staining was also performed to examine brain morphology.

\section{Statistics}

Subarachnoid hemorrhage grade, neurological scores, and beam balance scores were expressed as the median \pm 25th-75th percentiles and were analyzed by Mann-Whitney U-tests or Kruskal-Wallis tests, followed by SteelDwass multiple comparisons. Other values were expressed as the mean $\pm \mathrm{SD}$, and 1-way ANOVA with Tukey-Kramer post hoc tests were used. Mortality was compared using chi-square tests. A p value $<0.05$ was considered significant.

\section{Results}

\section{Deficiency of Endogenous TNC Prevents Brain Edema and BBB Disruption Following SAH}

Comparisons of physiological parameters revealed no significant differences among the groups (data not shown). None of 32 sham-operated mice died within the 48-hour observation period. The mortality of mice with SAH was lower in the TNKO group (22.6\%, 7 of 31 mice) than in the WT group (29.4\%, 10 of 34 mice), but the difference did not reach statistical significance. The median SAH grading score was similar between the WT and TNKO groups in each analysis at both 24 (10 vs 10.5 , respectively) and 48 (10 vs 9 , respectively) hours postsurgery. This indicated similar injury between the compared groups and suggested that differences in outcomes were the result of different treatments.

None of the groups exhibited neurological deficits before surgery. The WT SAH mice showed significantly worse neurological and beam balance scores compared with the sham-operated mice at 24 and 48 hours postsurgery. Neurological and beam balance scores in TNKO SAH mice were significantly better than in WT SAH mice at 24 hours postsurgery (Fig. 2). The TNKO SAH
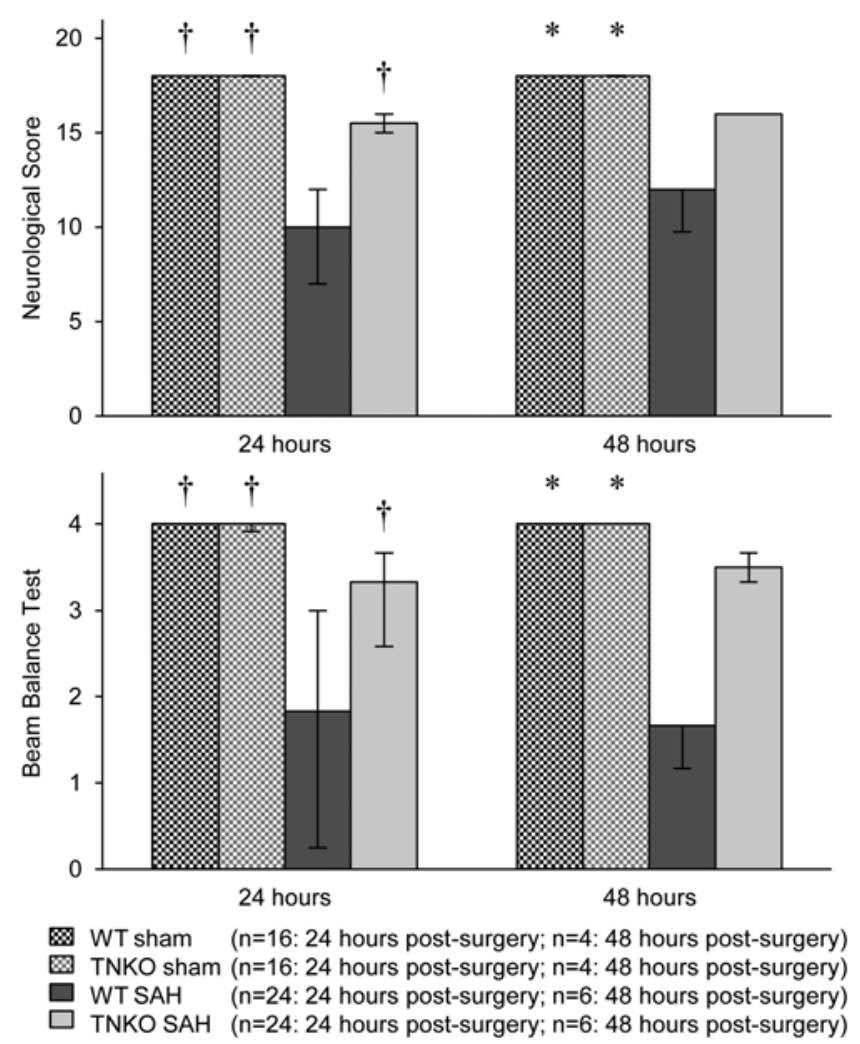

FIG. 2. Effects of endogenous TNC deficiency on neurological scores (upper) and beam balance test scores (lower) at 24 and 48 hours postsurgery. Data are expressed as the median \pm 25 th -75 th percentiles. ${ }^{*} p$ $<0.05, \uparrow p<0.01$ versus WT SAH group, Kruskal-Wallis tests. 

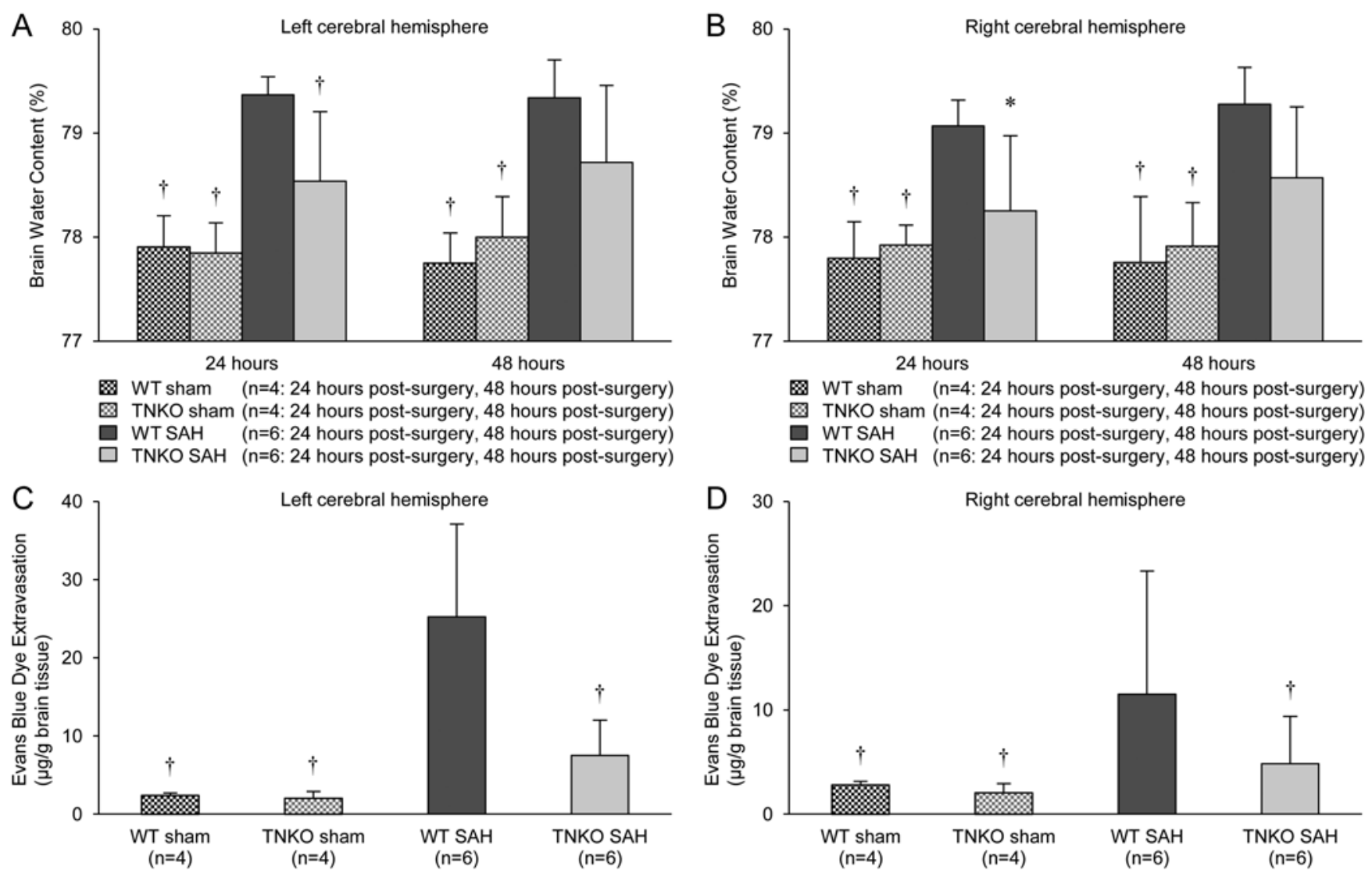

FIG. 3. Effects of endogenous TNC deficiency on brain water content ( $A$ and $B$ ) at 24 and 48 hours postsurgery, and Evans blue dye extravasation ( $C$ and $\mathbf{D}$ ) at 24 hours postsurgery. Data are expressed as the mean $\pm S D$. ${ }^{*} p<0.05, \uparrow p<0.01$ versus WT SAH group, ANOVA.

mice failed to improve their scores at 48 hours, but there was a trend toward improvement. The TNKO SAH mice showed neurological and beam balance scores similar to the sham-operated mice through the 48 -hour observation period.

Brain water content and Evans blue dye extravasation were similar between WT sham-operated and TNKO sham-operated mice. In WT mice, SAH resulted in a significant increase in brain water content at 24 and 48 hours postsurgery, and Evans blue dye extravasation at 24 hours postsurgery (Fig. 3). Compared with WT SAH mice, TNKO SAH mice showed significantly less brain water content and Evans blue dye extravasation at 24 hours postsurgery. There was no significant difference in brain water content and Evans blue dye extravasation between TNKO SAH and sham-operated mice through the observation period.

\section{Deficiency of Endogenous TNC Suppresses MMP-9 Induction and ZO-1 Degradation Following SAH}

Western blot analyses showed that the expression of TNC was weakly detected in WT sham-operated mice and was significantly upregulated in WT SAH mice. No TNC was detected in TNKO sham-operated and TNKO SAH mice (Fig. 4A and B). Expressions of MMP-9 and
ZO-1 were similar between WT sham-operated and TNKO sham-operated mice. SAH significantly increased MMP-9 levels and decreased ZO-1 levels in WT mice at 24 hours postsurgery. In TNKO SAH mice, MMP-9 induction and ZO-1 degradation were significantly suppressed compared with WT SAH mice (Fig. 4C and D).

\section{Deficiency of Endogenous TNC Inactivates MAPKs in Brain Capillary Endothelial Cells Following SAH}

Immunohistochemistry showed that phosphorylated ERK1/2, JNK, and p38 were minimally expressed in both WT and TNKO sham-operated mice and markedly increased in capillary endothelial cells in the temporal cortex in WT SAH mice at 24 hours post-SAH (Fig. 5). In TNKO SAH mice, however, phosphorylated ERK1/2, JNK, and p38 levels were remarkably suppressed and similar to sham-operated mice.

\section{Exogenous TNC Treatment Aggravates Brain Edema and BBB Disruption in TNKO SAH Mice}

Comparisons of physiological parameters revealed no significant differences among the groups (data not shown). None of 8 TNKO sham-operated mice with an injection of TNC died within the 24-hour observation period. The mortality following SAH was $20 \%$ (3 of 15 mice) in the 

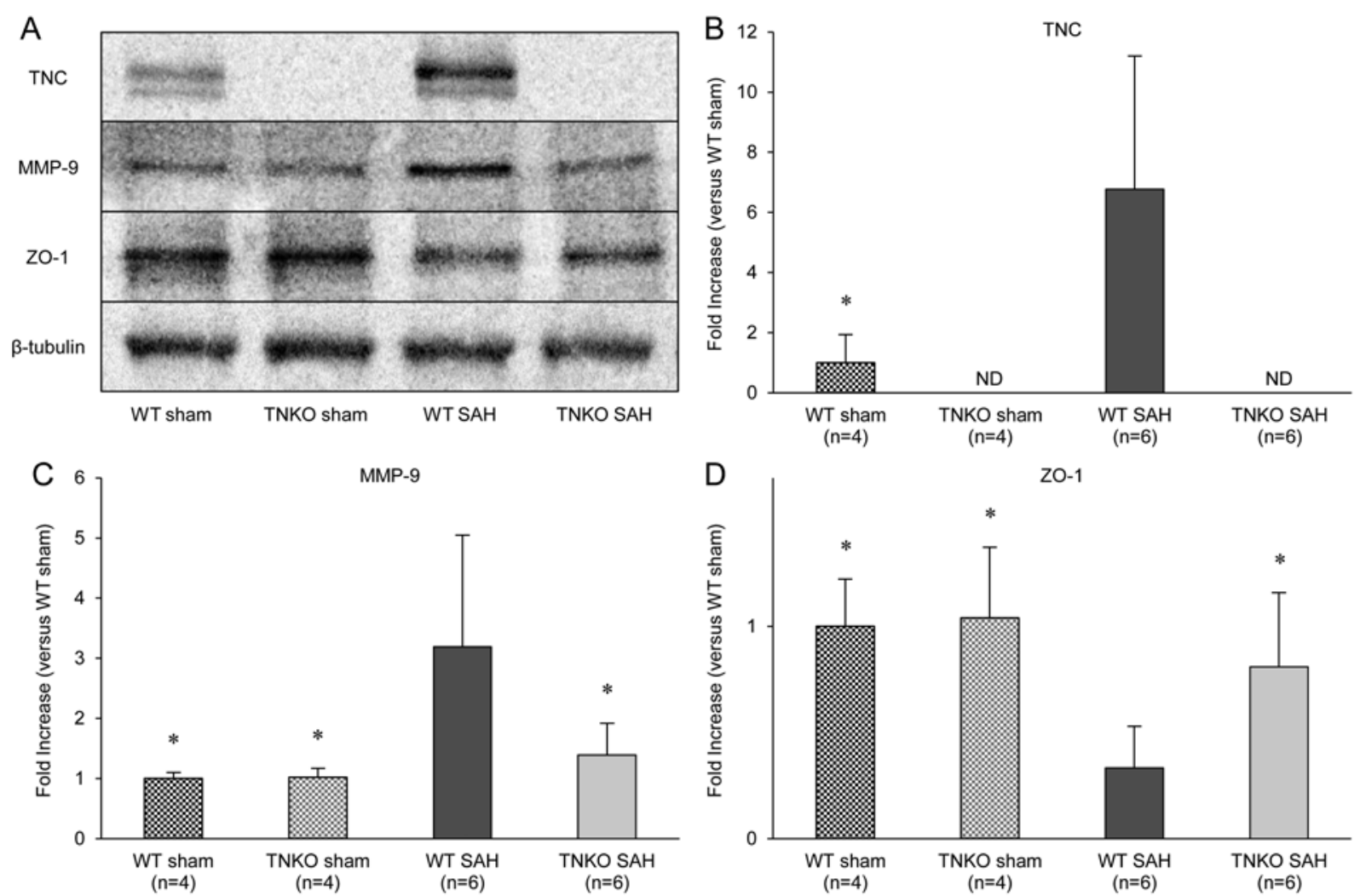

FIG. 4. Representative Western blots (A) and effects of endogenous TNC deficiency on expressions of TNC (B), MMP-9 (C), and ZO-1 (D) in the left cerebral hemisphere at 24 hours postsurgery. Expression levels of each protein are expressed as a ratio of $\beta$-tubulin levels for normalization and as the mean \pm SD. ND $=$ no detection. ${ }^{*} p<0.05$ versus WT SAH group, ANOVA.

PBS-injected group and $29.4 \%$ (5 of 17 mice) in the TNCinjected group. The median SAH grading score was similar between the groups (10.5 and 11 in the PBS-injected and TNC-injected groups, respectively), indicating that TNC treatment had no effect on SAH severity.

None of the mice exhibited neurological deficits before surgery. In TNKO sham-operated mice, TNC treatment had no effects on neuroscore, brain water content, and Evans blue dye extravasation. In TNKO SAH mice, TNC treatment aggravated neurological impairments, brain water content, and Evans blue dye extravasation compared with the PBS treatment (Figs. 6 and 7).

\section{Discussion}

In the present study, we investigated the role of TNC induction in brain edema formation and BBB disruption following SAH. Expression of TNC in the brain was weakly detected in WT sham-operated mice and was upregulated in WT SAH mice. TNKO suppressed neurological impairments, decreased brain water content, and Evans blue dye extravasation, and was associated with an inhibition of MMP-9 induction and the consequent preservation of the tight junction protein $\mathrm{ZO}-1$, as well as inactivation of MAPKs in brain capillary endothelial cells in mice with SAH. In TNKO sham-operated mice, exogenous TNC treatment had no effects on neuroscore, brain water content, and Evans blue dye extravasation. In contrast, in
TNKO SAH mice, exogenous TNC treatment aggravated these findings compared with PBS treatment. Thus, this study demonstrated that TNC plays an important role in the development of brain edema and BBB disruption following SAH.

Despite improvements in the clinical management of SAH, DCI remains one of the most important causes of morbidity and mortality in patients with SAH who survive the initial bleeding.${ }^{19}$ Recently, EBI, as well as delayed cerebral vasospasm, has been considered to be a cause of DCI. ${ }^{3}$ BBB disruption leading to brain edema formation has been involved in a key pathogenesis manifestation of EBI following experimental SAH. ${ }^{3}$ In a clinical setting as well, brain edema on CT was reported to be an independent risk factor for mortality and poor outcome following SAH. ${ }^{5}$ Many molecules, such as blood breakdown products and inflammatory mediators, may be involved, either acting simultaneously or at different stages during BBB disruption. ${ }^{20,21}$ To our knowledge, the present study demonstrated for the first time that TNC is an important causative factor for brain edema and BBB disruption, at least in an acute stage of SAH.

Expression of TNC, a matricellular protein, is extremely limited in the normal adult CNS, but is acutely induced following aneurysmal SAH. ${ }^{30-32}$ Higher CSF TNC levels were significantly associated with worse outcomes in a clinical setting. ${ }^{31}$ Experimental studies also reported that an excess of TNC expression might have harmful effects 


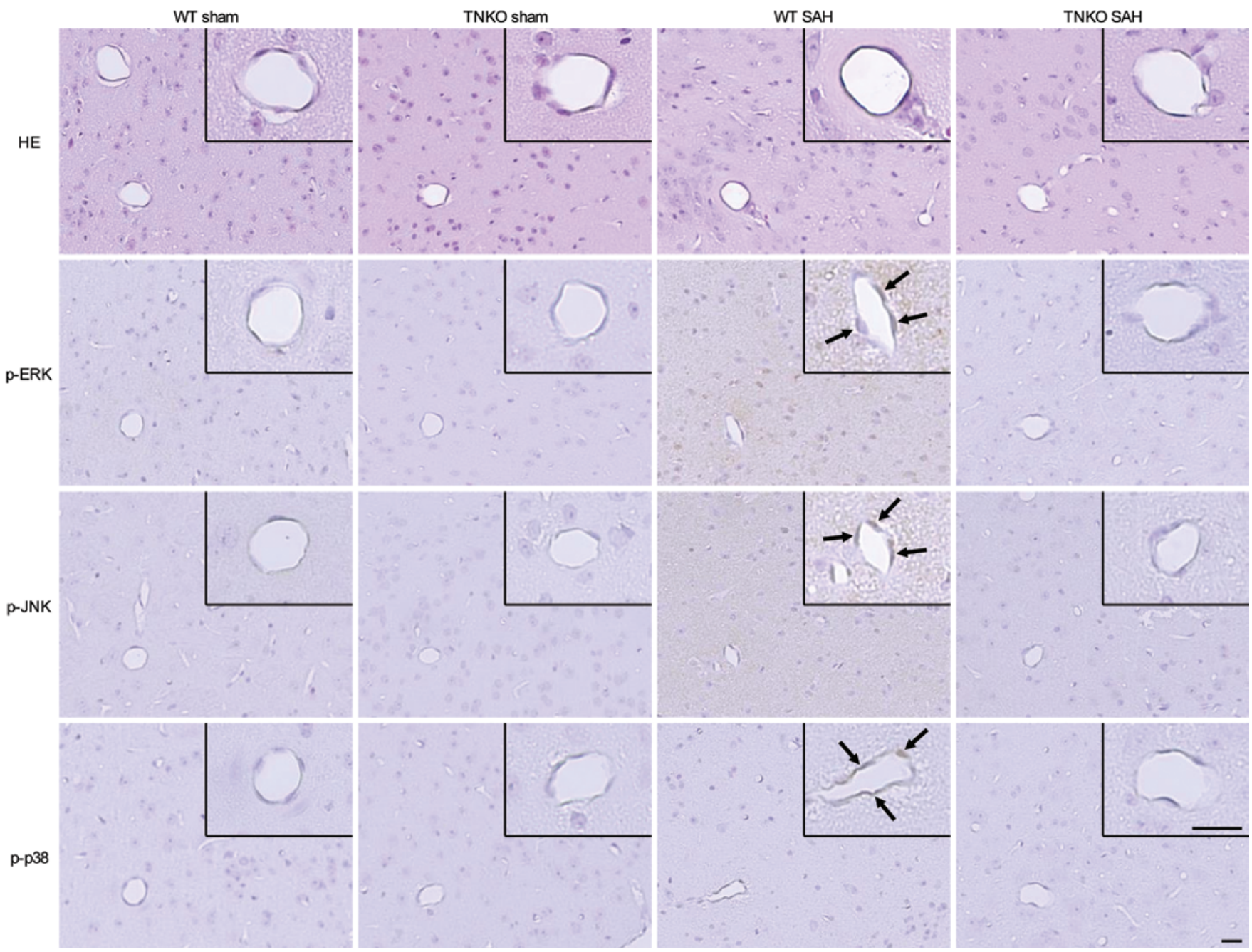

FIG. 5. Effects of endogenous TNC deficiency on immunohistochemical staining of phosphorylated ERK ( $p$-ERK) $1 / 2$, phosphorylated JNK (p-JNK), and phosphorylated p38 (p-p38) in the left temporal cortex at bregma $+1 \mathrm{~mm}$ at 24 hours postsurgery. $\mathrm{HE}=\mathrm{H}$ \& E staining. Arrows designate immunoreactive capillary endothelial cells. Insets show magnification of capillaries. Bar $=20 \mu \mathrm{m}$. Figure is available in color online only.

on carotid ${ }^{10}$ or cerebral arteries ${ }^{8,25}$ and the brain, ${ }^{24}$ possibly by activating MAPKs. Because platelet-derived growth factor (PDGF) is a well-known inducer of TNC, imatinib mesylate, an inhibitor of the tyrosine kinases of PDGF receptors, was used to inhibit TNC upregulation following experimental SAH. ${ }^{24,25}$ As a result, imatinib mesylate prevented both cerebral vasospasm and neuronal apoptosis by inactivating MAPK in SAH rats, improving neurological function. ${ }^{24,25}$ However, the possibility that imatinib exerted the neuroprotective effects by mechanisms unrelated to TNC could not be excluded. Thus far, neither clinical nor experimental studies have investigated the possible relationships between TNC expression and brain edema formation or BBB disruption.

Accumulated evidence supports a role for MMP-9 in BBB disruption following SAH., ${ }^{711,22,23,28}$ MMP-9 levels in CSF and peripheral blood were significantly increased in patients with SAH..$^{11,22}$ MMP-9 degrades the extracellular matrix proteins of cerebral microvessel basal lamina and interendothelial tight junction proteins such as ZO-1, causing BBB disruption and brain edema formation. ${ }^{23,28}$ A recent study showed that MMP-9 knockout inhibited post-SAH brain edema formation in mice. Inflammatory cytokines and other factors can regulate the transcription of MMP-9 following SAH. ${ }^{20,28}$ TNC was also reported to upregulate MMP-9 expression in cultured cancer cells. ${ }^{12,14}$ In addition, TNKO mice showed a marked decrease in expressions of inflammatory cytokines and MMP-9 after hepatic ischemia and reperfusion. ${ }^{15}$ This study first demonstrated the link between TNC induction and MMP-9 upregulation or BBB disruption in the brain following $\mathrm{SAH}$ by using TNKO mice and exogenous TNC treatment. Although it remains unknown how TNC upregulates MMP9, TNC induces MAPKs, ${ }^{8,15}$ which are known to upregulate MMP-9. ${ }^{4}$ Because this study also demonstrated that TNC regulated MAPK activation in brain capillary endothelial cells, it may be reasonable to consider that TNC induces MMP-9 and causes BBB disruption in mice with SAH via MAPK activation.

Another interesting finding in this study is that exoge- 

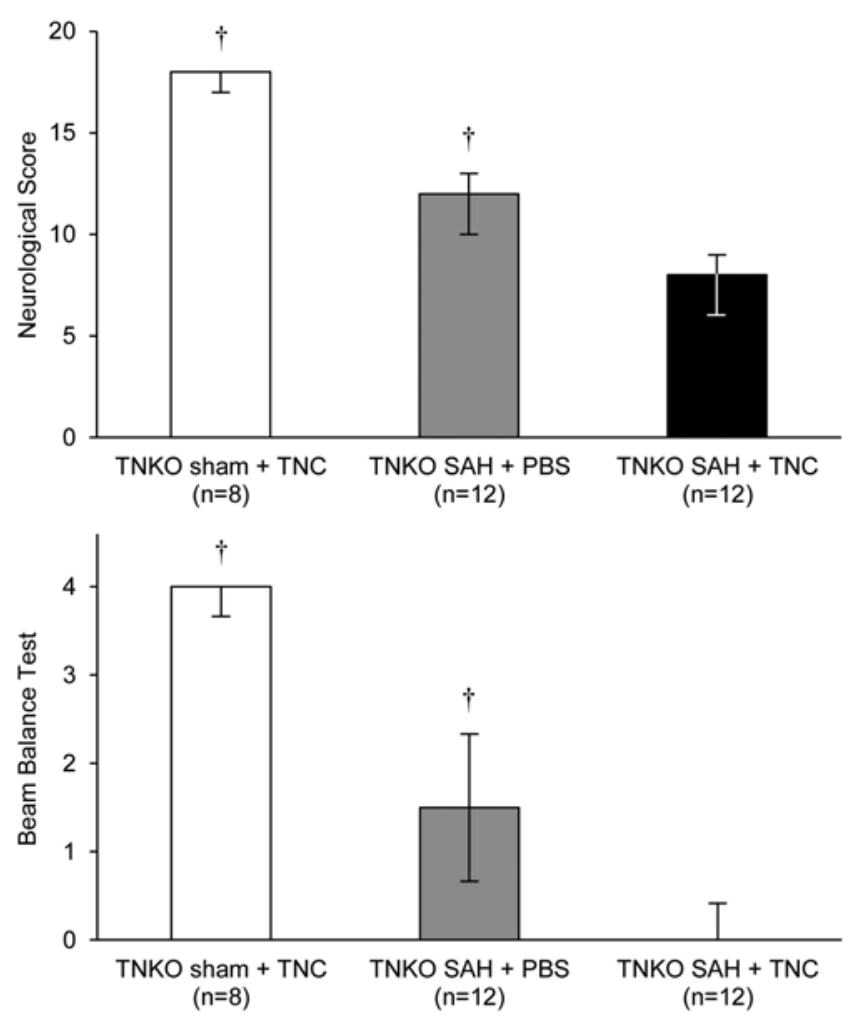

FIG. 6. Effects of exogenous TNC treatment on neurological scores (upper) and beam balance test scores (lower) in TNKO mice at 24 hours postsurgery. Data are expressed as the median \pm 25 th -75 th percentiles. $t p<0.01$ versus TNKO SAH + TNC group, Kruskal-Wallis tests. nous TNC treatment aggravated BBB disruption in TNKO SAH mice, but had no effects on TNKO sham-operated mice. The possible explanation is that intact TNC-induced signaling is different from post-SAH TNC signaling because SAH induces MMP-9 and other MMPs, which can cleave TNC.${ }^{16,34}$ For example, the cleaved products (including epidermal growth factor-like domains of TNC) caused apoptosis of cultured vascular smooth muscle cells, whereas intact TNC had no proapoptotic effects. ${ }^{34}$ Thus, postSAH TNC induction upregulates MMP-9, which in turn cleaves TNC, possibly activating different pathways that can cause brain injuries.

This study has limitations. First, 1 dose of exogenous TNC was administered only to TNKO SAH mice to confirm that TNC deficiency suppressed BBB disruption. If this study had shown the dose-dependent aggravation of TNC on BBB disruption in TNKO SAH mice or the aggravation of $\mathrm{BBB}$ disruption by exogenous TNC treatment in WT SAH mice, the role of TNC in BBB disruption would have been strengthened. Second, this study showed MMP-9 induction as one of several possible mechanisms for post-SAH TNC-induced BBB disruption. However, we cannot exclude the possibility that TNC caused BBB disruption by mechanisms unrelated to MMP-9. Thus, the possible involvement of other actions of TNC needs to be examined.

\section{Conclusions}

In this study, we showed evidence for TNC to cause brain edema and BBB disruption following $\mathrm{SAH}$ in mice,
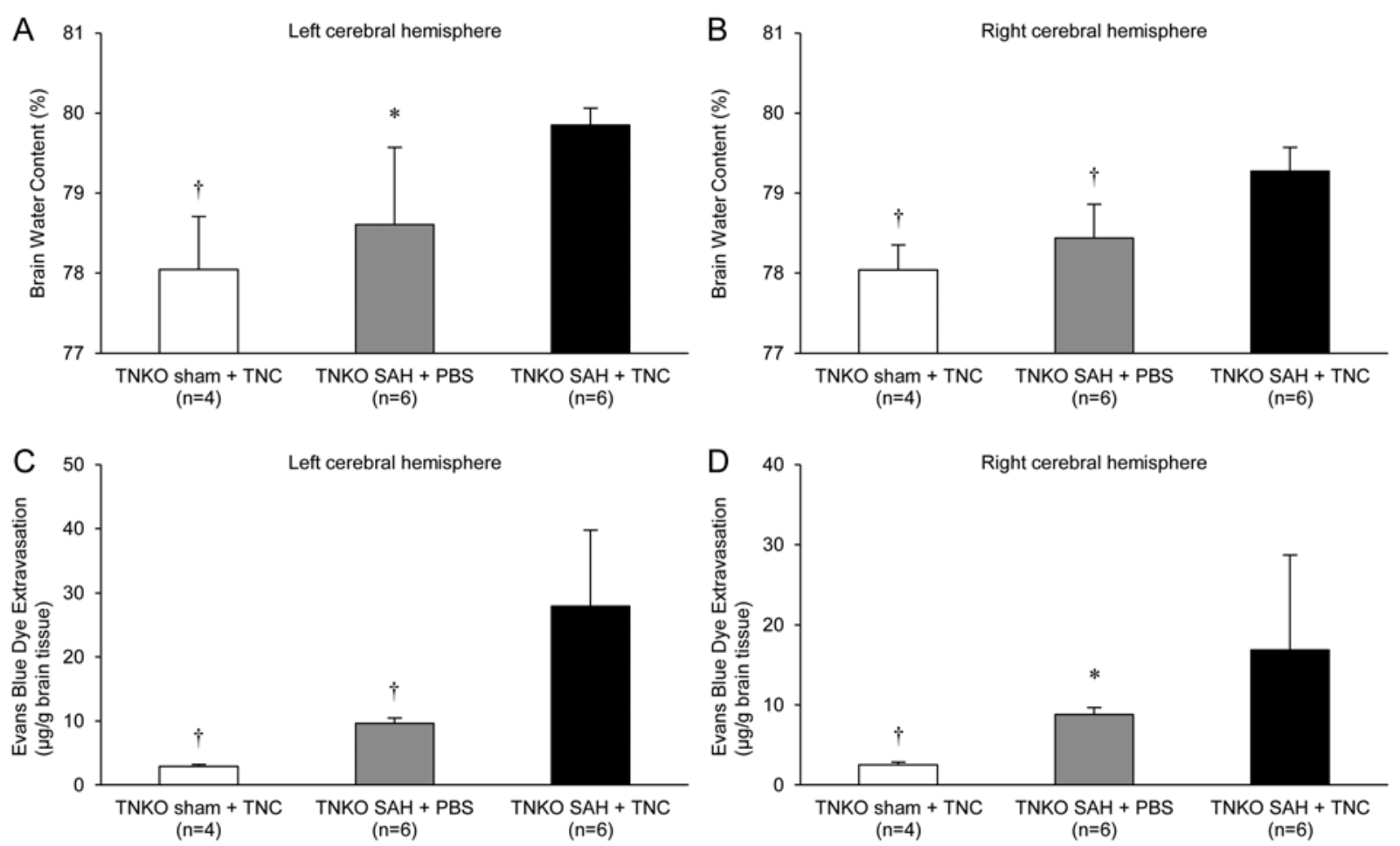

FIG. 7. Effects of exogenous TNC treatment on brain water content ( $A$ and $B$ ) and Evans blue dye extravasation (C and D) in TNKO mice at 24 hours postsurgery. Data are expressed as the mean \pm SD. ${ }^{*} p<0.05, \uparrow p<0.01$ versus TNKO SAH + TNC group, ANOVA. 
possibly by MAPK-mediated MMP-9 induction and ZO-1 degradation. TNC could be a molecular target against which to develop new therapies for SAH-induced brain injuries.

\section{Acknowledgments}

This work was supported in part by a grant-in-aid for Scientific Research from the Mie Society for the Promotion of Medical Research to Dr. Fujimoto, and the Japan Society for the Promotion of Science to Drs. Shiba, Fujimoto, and Suzuki. We thank Ms. Chiduru Yamamoto (Department of Neurosurgery, Mie University Graduate School of Medicine) and Ms. Mari Hara (Department of Pathology and Matrix Biology, Mie University Graduate School of Medicine) for their technical assistance.

\section{References}

1. Altay O, Suzuki H, Hasegawa Y, Caner B, Krafft PR, Fujii $\mathrm{M}$, et al: Isoflurane attenuates blood-brain barrier disruption in ipsilateral hemisphere after subarachnoid hemorrhage in mice. Stroke 43:2513-2516, 2012

2. Ayer RE, Sugawara T, Chen W, Tong W, Zhang JH: Melatonin decreases mortality following severe subarachnoid hemorrhage. J Pineal Res 44:197-204, 2008

3. Caner B, Hou J, Altay O, Fuj M II, Zhang JH: Transition of research focus from vasospasm to early brain injury after subarachnoid hemorrhage. J Neurochem 123 (Suppl 2):1221,2012

4. Cho A, Graves J, Reidy MA: Mitogen-activated protein kinases mediate matrix metalloproteinase- 9 expression in vascular smooth muscle cells. Arterioscler Thromb Vasc Biol 20:2527-2532, 2000

5. Claassen J, Carhuapoma JR, Kreiter KT, Du EY, Connolly ES, Mayer SA: Global cerebral edema after subarachnoid hemorrhage: frequency, predictors, and impact on outcome. Stroke 33:1225-1232, 2002

6. Eroglu C: The role of astrocyte-secreted matricellular proteins in central nervous system development and function. $\mathbf{J}$ Cell Commun Signal 3:167-176, 2009

7. Feiler S, Plesnila N, Thal SC, Zausinger S, Schöller K: Contribution of matrix metalloproteinase-9 to cerebral edema and functional outcome following experimental subarachnoid hemorrhage. Cerebrovasc Dis 32:289-295, 2011

8. Fujimoto M, Suzuki H, Shiba M, Shimojo N, ImanakaYoshida K, Yoshida T, et al: Tenascin-C induces prolonged constriction of cerebral arteries in rats. Neurobiol Dis 55:104-109, 2013

9. Golledge J, Clancy P, Maguire J, Lincz L, Koblar S: The role of tenascin $\mathrm{C}$ in cardiovascular disease. Cardiovasc Res 92:19-28, 2011

10. Hamada K, Miura Y, Toma N, Miyamoto K, Imanaka-Yoshida K, Matsushima S, et al: Gellan sulfate core platinum coil with tenascin-C promotes intra-aneurysmal organization in rats. Transl Stroke Res 5:595-603, 2014

11. Horstmann S, Su Y, Koziol J, Meyding-Lamadé U, Nagel S, Wagner S: MMP-2 and MMP-9 levels in peripheral blood after subarachnoid hemorrhage. J Neurol Sci 251:82-86, 2006

12. Ilunga K, Nishiura R, Inada H, El-Karef A, Imanaka-Yoshida K, Sakakura T, et al: Co-stimulation of human breast cancer cells with transforming growth factor-beta and tenascin-C enhances matrix metalloproteinase-9 expression and cancer cell invasion. Int J Exp Pathol 85:373-379, 2004

13. Imanaka-Yoshida $\mathrm{K}$ : Tenascin- $\mathrm{C}$ in cardiovascular tissue remodeling: from development to inflammation and repair. Circ J 76:2513-2520, 2012

14. Kalembeyi I, Inada H, Nishiura R, Imanaka-Yoshida K, Sakakura T, Yoshida T: Tenascin-C upregulates matrix metalloproteinase- 9 in breast cancer cells: direct and synergistic effects with transforming growth factor beta1. Int J Cancer 105:53-60, 2003

15. Kuriyama N, Duarte S, Hamada T, Busuttil RW, Coito AJ: Tenascin-C: a novel mediator of hepatic ischemia and reperfusion injury. Hepatology 54:2125-2136, 2011

16. Mehta V, Russin J, Spirtos A, He S, Adamczyk P, Amar AP, et al: Matrix metalloproteinases in cerebral vasospasm following aneurysmal subarachnoid hemorrhage. Neurol Res Int 2013:943761, 2013

17. Midwood KS, Orend G: The role of tenascin-C in tissue injury and tumorigenesis. J Cell Commun Signal 3:287-310, 2009

18. Nag S, Manias JL, Stewart DJ: Pathology and new players in the pathogenesis of brain edema. Acta Neuropathol 118:197-217, 2009

19. Rowland MJ, Hadjipavlou G, Kelly M, Westbrook J, Pattinson KT: Delayed cerebral ischaemia after subarachnoid haemorrhage: looking beyond vasospasm. Br J Anaesth 109:315-329, 2012

20. Sabri M, Lass E, Macdonald RL: Early brain injury: a common mechanism in subarachnoid hemorrhage and global cerebral ischemia. Stroke Res Treat 2013:394036, 2013

21. Saga Y, Yagi T, Ikawa Y, Sakakura T, Aizawa S: Mice develop normally without tenascin. Genes Dev 6:1821-1831, 1992

22. Sarrafzadeh A, Copin JC, Bengualid DJ, Turck N, Vajkoczy P, Bijlenga P, et al: Matrix metalloproteinase- 9 concentration in the cerebral extracellular fluid of patients during the acute phase of aneurysmal subarachnoid hemorrhage. Neurol Res 34:455-461, 2012

23. Sehba FA, Mostafa G, Knopman J, Friedrich V Jr, Bederson JB: Acute alterations in microvascular basal lamina after subarachnoid hemorrhage. J Neurosurg 101:633-640, 2004

24. Shiba M, Fujimoto M, Imanaka-Yoshida K, Yoshida T, Taki W, Suzuki H: Tenascin-C causes neuronal apoptosis after subarachnoid hemorrhage in rats. Transl Stroke Res 5:238247, 2014

25. Shiba M, Suzuki H, Fujimoto M, Shimojo N, Imanaka-Yoshida K, Yoshida T, et al: Imatinib mesylate prevents cerebral vasospasm after subarachnoid hemorrhage via inhibiting tenascin-C expression in rats. Neurobiol Dis 46:172-179, 2012

26. Suarez JI, Tarr RW, Selman WR: Aneurysmal subarachnoid hemorrhage. N Engl J Med 354:387-396, 2006

27. Sugawara T, Ayer R, Jadhav V, Zhang JH: A new grading system evaluating bleeding scale in filament perforation subarachnoid hemorrhage rat model. J Neurosci Methods 167:327-334, 2008

28. Suzuki H, Ayer R, Sugawara T, Chen W, Sozen T, Hasegawa $\mathrm{Y}$, et al: Protective effects of recombinant osteopontin on early brain injury after subarachnoid hemorrhage in rats. Crit Care Med 38:612-618, 2010

29. Suzuki H, Hasegawa Y, Kanamaru K, Zhang JH: Mechanisms of osteopontin-induced stabilization of blood-brain barrier disruption after subarachnoid hemorrhage in rats. Stroke 41:1783-1790, 2010

30. Suzuki H, Kanamaru K, Shiba M, Fujimoto M, ImanakaYoshida K, Yoshida T, et al: Cerebrospinal fluid tenascin-C in cerebral vasospasm after aneurysmal subarachnoid hemorrhage. J Neurosurg Anesthesiol 23:310-317, 2011

31. Suzuki H, Kanamaru K, Suzuki Y, Aimi Y, Matsubara N, Araki T, et al: Tenascin-C is induced in cerebral vasospasm after subarachnoid hemorrhage in rats and humans: a pilot study. Neurol Res 32:179-184, 2010

32. Suzuki H, Kinoshita N, Imanaka-Yoshida K, Yoshida T, Taki $\mathrm{W}$ : Cerebrospinal fluid tenascin-C increases preceding the development of chronic shunt-dependent hydrocephalus after subarachnoid hemorrhage. Stroke 39:1610-1612, 2008

33. van Gijn J, Kerr RS, Rinkel GJ: Subarachnoid haemorrhage. Lancet 369:306-318, 2007 
34. Wallner K, Li C, Shah PK, Wu KJ, Schwartz SM, Sharifi BG: EGF-Like domain of tenascin-C is proapoptotic for cultured smooth muscle cells. Arterioscler Thromb Vasc Biol 24:1416-1421, 2004

35. Yoshida T, Yoshimura E, Numata H, Sakakura Y, Sakakura $\mathrm{T}$ : Involvement of tenascin- $\mathrm{C}$ in proliferation and migration of laryngeal carcinoma cells. Virchows Arch 435:496-500, 1999

36. Zappaterra MW, LaMantia AS, Walsh CA, Lehtinen MK: Isolation of cerebrospinal fluid from rodent embryos for use with dissected cerebral cortical explants. J Vis Exp 73:e50333, 2013

\section{Disclosure}

Prof. T. Yoshida has received a royalty from IBL-Japan on antibodies against tenascin-C.

\section{Author Contributions}

Conception and design: Suzuki, Imanaka-Yoshida, Yoshida. Acquisition of data: Fujimoto, Shiba, Kawakita, Liu, Shimojo. Analysis and interpretation of data: all authors. Drafting the article: Suzuki, Fujimoto. Critically revising the article: all authors. Reviewed submitted version of manuscript: all authors. Approved the final version of the manuscript on behalf of all authors: Suzuki. Statistical analysis: Fujimoto, Shiba, Kawakita, Liu, Shimojo, Imanaka-Yoshida, Yoshida. Administrative/technical/material support: Imanaka-Yoshida, Yoshida. Study supervision: Suzuki, Yoshida.

\section{Correspondence}

Hidenori Suzuki, Department of Neurosurgery, Mie University Graduate School of Medicine, 2-174 Edobashi, Tsu, Mie 5148507, Japan. email: mie1192suzuki@gmail.com. 